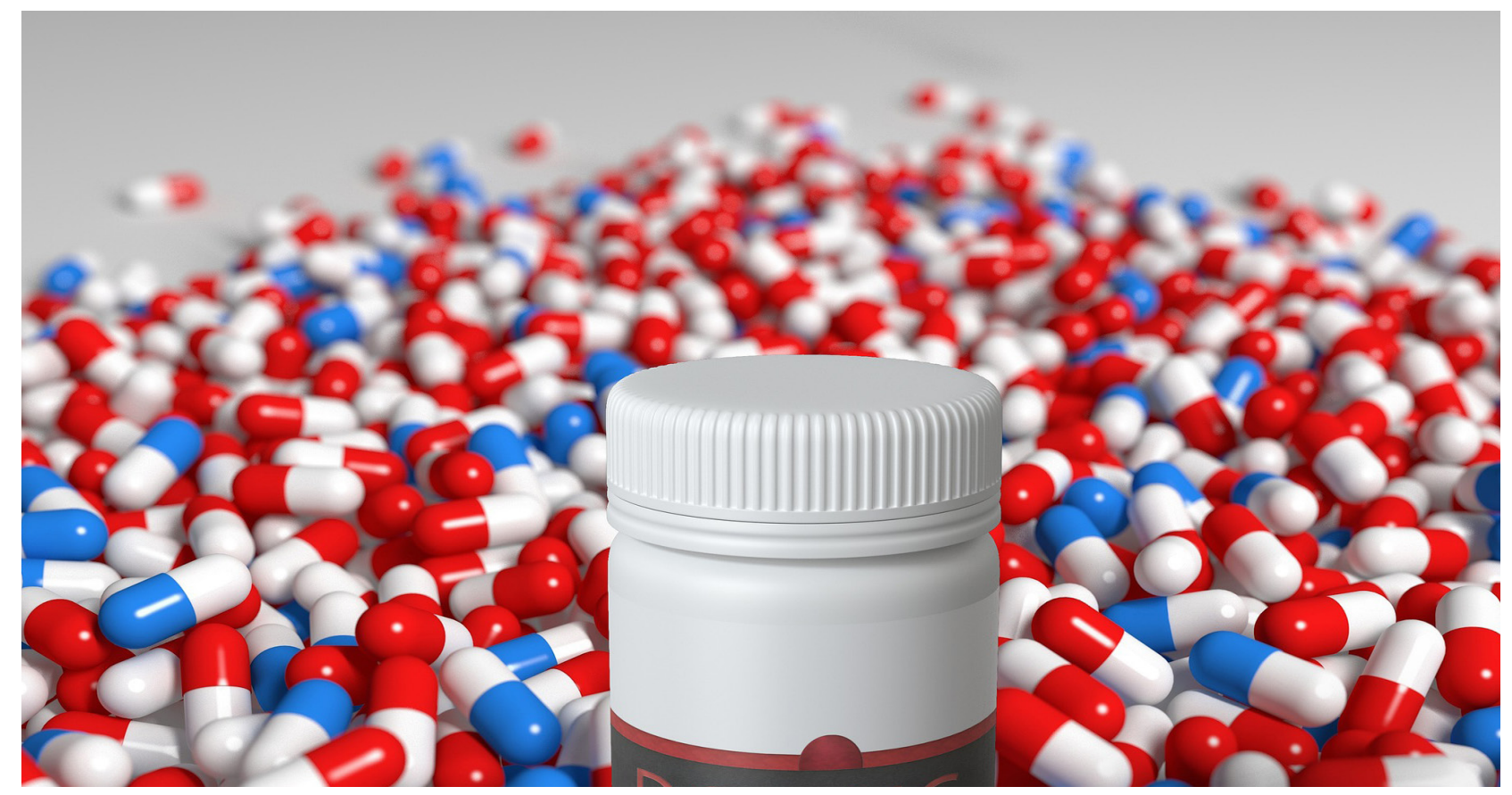

\title{
Propuesta de prevención del consumo de sustancias psicoactivas en los jóvenes desde un enfoque comunitario
}

\section{Proposal for the prevention of consumption of psychoactive substances by young people from a community approach}

Luis Oscar Barroso-Carrillo

Especialista en Gestión Pública, Gp_lo_barroso@fesc.edu.co https://orcid.org/0000-0002-1847-8542,

Fundación de Estudios Superiores Comfanorte, Cúcuta, Colombia

Maria Alejandra Benitez-Hurtado

Magíster en Derecho Administrativo, maria.benitez@unitecnar.edu.co

https://orcid.org/0000-0001-5546-3000 Fundación Universitaria Antonio de Arevalo - Unitecnar, Cartagena de Indias, Colombia 


\section{6}

Recibido: Junio 22 de 2019

Aceptado: Noviembre 20 de 2019

Cómo citar: L.O Barroso-Carrillo y M.A Benitez-Hurtado, "Propuesta de prevención del consumo de sustancias psicoactivas en los jóvenes

desde un enfoque comunitario", Sostenibilidad, Tecnología y Humanismo, vol. 11, no. 1, 65-78, 2020

Resumen

El objetivo del trabajo fue plantear una propuesta de gestión pública para la prevención del consumo de sustancias psicoactivas en jóvenes, teniendo en cuenta conceptos del Capital Social, Capital Intelectual y a través de un diseño metodológico de corte cuantitativo, observacional, descriptivo y documental; utilizando un instrumento ad hoc aplicado a través de una entrevista online a 341 jóvenes del municipio de Toledo. Se analizaron aspectos del entorno social y familiar, capacidad de influencia de las roscas y conductas parentales, en las decisiones de los jóvenes frente al consumo. Como resultado se encontró que existe tendencia hacia relaciones de amistad con consumidores de alcohol, alto porcentaje de consumo de bebidas alcohólicas en hogares, facilidad para acceder a sustancias psicoactivas y un rango de edad promedio de su primera experiencia de consumo entre 13 y 16 años. Lo anterior, abre discusión sobre inexistencia de programas sociales que involucren la juventud, adecuado control parental e ineficacia de las estrategias preventivas. Se concluye que la implementación de un modelo de gestión pública de prevención basado en enfoque comunitario y caracterizado por cambiar el entorno social, será una importante herramienta que ayude al fortalecimiento del capital social, generador de desarrollo integral y bienestar.

Palabras clave: Propuesta, prevención, gestión pública, jóvenes, sustancias psicoactivas.
Abstract

The objective of the work was to propose a public management proposal for the prevention of psychoactive substance consumption in young people, taking into account concepts of Social Capital, Intellectual Capital and through a quantitative, observational, descriptive and documentary methodological design; using an ad hoc instrument applied through a online interview to 341 young people from the municipality of Toledo. Aspects of the social and family environment, capacity of influence of the threads and parental behaviors, in the decisions of the young people in front of the consumption were analyzed. As a result, it was found that there is a tendency towards friendly relationships with alcohol consumers, a high percentage of alcoholic beverage consumption in homes, ease of access to psychoactive substances and an average age range of their first consumer experience between 13 and 16 years old. This opens a discussion about the lack of social programs involving youth, adequate parental control and ineffective preventive strategies. It is concluded that the implementation of a public management model of prevention based on a community approach and characterized by changing the social environment, will be an important tool to help strengthen social capital, which generates comprehensive development and welfare.

Keywords: Proposal, prevention, public management, youth, psychoactive substances. 
Introducción

El consumo de sustancias psicoactivas es una situación que afecta a todas las regiones del mundoya pesardelas múltiples investigaciones y acciones, este sigue generando impacto negativo en las sociedades modernas de todos los Estados, afectando particularmente a los jóvenes, quienes por su condición de desarrollo presentan una considerable vulnerabilidad. En consecuencia, los estudios realizados por diversas Instituciones corroboran el sombrío panorama al que se ve enfrentado el mundo actual e insisten que mientras no exista una verdadera voluntad política de los gobiernos, es difícil mostrar resultados positivos en materia de prevención y control del consumo [1].

En este orden, un estudio sobre el consumo de drogas en las Américas, enfatiza que en la región sigue siendo una situación altamente sensible y que se continúa desafiando a los formuladores de políticas públicas basadas en la cooperación internacional en materia de prevención [2]. Resalta el mencionado informe, el inicio del consumo a edades tempranas y la relación de este con la disminución del rendimiento escolar, aumento de conflictos y prácticas sexuales riesgosas. En consecuencia, se evidencia la estrecha relación existente entre consumo, violencia y los efectos producidos en el entorno del individuo afectado [3].

Así mismo, en el último informe la OPS [4] acentúa que el alcoholismo aumenta la desigualdad entre las personas y por ende entre los países, afectando negativamente el logro de las metas propuestas en el Objetivo de Desarrollo Sostenible número diez, que propone la ONU en su agenda 2030 respecto a la lucha por la disminución de las brechas de desigualdad social, lo que hace que el problema no solamente sea social, sino también económico.
El municipio de Toledo (Norte de Santander), no es ajeno a la presencia de este flagelo que afecta negativamente el desarrollo de la juventud, acentuándose la problemática debido a las condiciones sociales existentes, incluyendo los efectos del conflicto armado que por años ha afectado los municipios distantes del País [5]. Por tal motivo, es importante dar respuesta a la pregunta: cómo o cual será el modelo de gestión adecuado que permita mitigar el consumo de SPA en los jóvenes del municipio y así, plantear una propuesta de gestión pública para la prevención del consumo de SPA en los jóvenes. La cual, deberá estar conformada por múltiples estrategias que ayuden a la formación y construcción de seres humanos competentes en los aspectos sociales y culturales, a fin de que puedan enfrentar con mayor facilidad el futuro que les espera [6].

Esta investigación pretende generar una propuesta que garantice a la juventud un desarrollo adecuado que contribuya a la formación de un apropiado capital social para que en el futuro sean dignos transformadores de conocimiento en fuente de riqueza tanto para sus familias como para la comunidad [7]; pues es evidente que el Capital Social está estrechamente relacionado con la consolidación del tejido social y se construye principalmente a partir de la confianza que exista entre las sociedades, inclusive por encima de los elementos que conforman la democracia [8].

Es necesario promover la articulación en las instituciones a fin de lograr los objetivos propuestos y aprovechar las capacidades propias de cada una [9] y así promover una economía del conocimiento que permita delimitar una ruta adecuada hacia el desarrollo social. Por lo tanto y de acuerdo a lo revelado por [10] sobre el desconocimiento que poseen los gerentes públicos respecto de la gestión basada en el conocimiento, se hace necesario 


\section{8}

plantear propuestas que involucren a múltiples instituciones en el logro de objetivos comunes. Se tendrán en cuenta modelos que generen credibilidad y empoderamiento, es por esta razón que se darán prioridad a los modelos basados en la comunidad, en donde se tenga en cuenta la opinión y acción de los involucrados [11].

Igualmente, es importante anotar que los procesos de gestión pública tratan elementos particulares para cada Estado [12], los cuales buscan garantizar la eficiencia y calidad mediante la aplicación de modelos integrados que han sido diseñados y adoptados tras años de investigación [13]. Es necesario aplicar las metodologías requeridas en base a la realidad social encontrada; pero sin olvidar la participación ciudadana en el diseño, gestión y ejecución de la política dentro del marco normativo existente [14]. Es necesario la consolidación de equipos interdisciplinarios con profesionales diversos y comunidad, ya que la contribución multidisciplinaria contribuye al fortalecimiento académico tanto del gerente o gestor público como de los profesionales que forman parte del equipo investigativo para los fines determinados [15]. Lo anterior, debido a que las Organizaciones pueden adquirir calidad en sus procesos mediante la mejora de sus conocimientos dentro de la misma institución, aplicando estratégicamente estas nuevas técnicas adquiridas, lo cual además aportará valor a sus intangibles [16].

Materiales y métodos

El modelo de investigación descritptivocuantitativo, basado en la observación y descripción se evaluaron los resultados de un instrumento aplicado, teniendo en cuenta que los estudios observacionales son los más usados en las ciencias sociales para investigar temas de prevención. En consecuencia, se plantea una propuesta de gestión pública para la prevención del consumo de sustancias psicoactivas en los jovenes, la cual tiene como novedad, un enfoque de movilización hacia la comunidad; en donde las Organizaciones tanto públicas como privadas se vinculen bajo principios de creación de valor compartido dentro del ámbito de la responsabilidad social [17].

El enfoque del estudio es documental, por cuanto se tuvieron en cuenta, libros, documentos y artículos que aportaron información sobre el tema y que permitieron examinar modelos exitosamente aplicados y evaluados.

La población de jóvenes considerada para la investigación fue entre edades de 10 y 19 años del municipio de Toledo, para lo cual se recurre a Dirección Nacional de Planeación [18] a través de TerriData, que es una herramienta usada para el fortalecimiento de la gestión pública, cuyo objetivo es promover el uso de indicadores estandarizados y comparables. Entonces, haciendo uso de la mencionada herramienta se accede al indicador de Censo 2005 y proyecciones DANE, para obtener de allí la información requerida, la cual se resume en la tabla 1.

Tabla 1. Cálculo proyecciones habitantes entre 10 y 19 años Municipio de Toledo (año 2019)

\begin{tabular}{ll}
\hline Grupo Poblacional & Año 2019 \\
Mujeres entre $10 \mathrm{y} 19$ años & 1.506 \\
Hombres entre $10 \mathrm{y} 19$ años & 1.549 \\
& \\
Tulal & 3055
\end{tabular}

A continuación en la tabla 2 se puede observar el cáculo de la muestra para el presente estudio, el cual arrojó un total de 341 jóvenes a quienes se les aplicaron los instrumentos definidos. 
Tabla 2. Cálculo del tamaño de la muestra.

\begin{tabular}{lll}
\hline Fórmula & Reemplazo de datos & Resultado (tamaño de la muestra) \\
\hline$\frac{N \times\left(a_{c} * 0.5\right)^{2}}{1+\left(e^{2} *(N-1)\right.}$ & $\frac{30.55 *(1.96 * 0.5)^{2}}{1+\left(0.05^{2} *(3055-1)\right.}$ & 341 \\
\hline
\end{tabular}

Como instrumento se utilizó un cuestionario tipo Likert, más utilizado en el campo de la investigación del trabajo social. También se implementó una encuesta que se desarrolló teniendo como referencia algunos items aplicados en el estudio de 2016 sobre consumo de SPA en escolares [19].

\section{Resultados}

Con el fin de indagar sobre los antecedentes existentes referentes al consumo de SPA en el municipio de Toledo, se recurre a sintetizar y deducir para hallar las consecuencias, partiendo desde un supuesto y aplicando razonamientos desde lo general hasta lo particular. En consecuencia, se observa y describen los antecedentes globales que presentan los informes de la Oficina de las Naciones Unidas contra la Droga y el Delito [1] los cuales evidencian incremento del consumo. Los informes de la Comisión interamericana para el control y abuso de drogas [2], los cuales resaltan a países como Colombia, Chile, Argentina y Uruguay con un preocupante incremento en el consumo de sustancias psicoactivas. En el plano nacional, el Observatorio de Drogas Colombia [20] evidencia un incremento de alrededor del $70 \%$ en el consumo de alcohol sobre todo en estudiantes de secundaria.

A nivel local, se evidencia la falta de estudios focalizados al respecto, esto generalmente es consecuencia del conflicto armado que por años ha afectado a Colombia y que de alguna manera desestimula la investigación en las regiones apartadas [5]. Lo anteriormente planteado y otros factores como la ausencia de docentes en escuelas rurales, la presencia de empresas mineras foráneas y la falta de control parental, han limitado oportunidades de desarrollo y abren espacios para que el problema del aumento del consumo se incrementen en los jóvenes del municipio de Toledo [21].

Una vez aplicados los instrumentos definidos se obtuvieron los siguientes resultados:

El primer hallazgo importante es que la mayoría de los jóvenes encuestados (33\%) tienen amigos que han consumido bebidas embriagantes repetidas veces, un $32 \%$ de esos amigos casi nunca lo hace y solo el $19 \%$ nunca lo hace; pero el $15 \%$ de los encuestados si tienen amigos que consumen bebidas embriagantes continuamente, en este orden, $11 \%$ de los encuestados manifestaron que siempre hay amigos dentro de su grupo selecto de amistades que si son consumidores de SPA. De otro lado, alrededor del 39\% de los encuestados han consumido repetidas veces SPA (licor, cigarrillo, alucinógenos, etc), el $27 \%$ casi nunca lo ha hecho y el $18 \%$ nunca lo ha hecho; pero aproximadamente el $16 \%$ manifestaron que siempre lo hacen. Igualmente, se evidencia que alrededor del $68 \%$ de los encuestados manifestó que no hay personas alcohólicas en sus respectivos hogares y el $32 \%$ de los encuestados manifiesta que si existen personas alcohólicas en sus respectivos hogares.

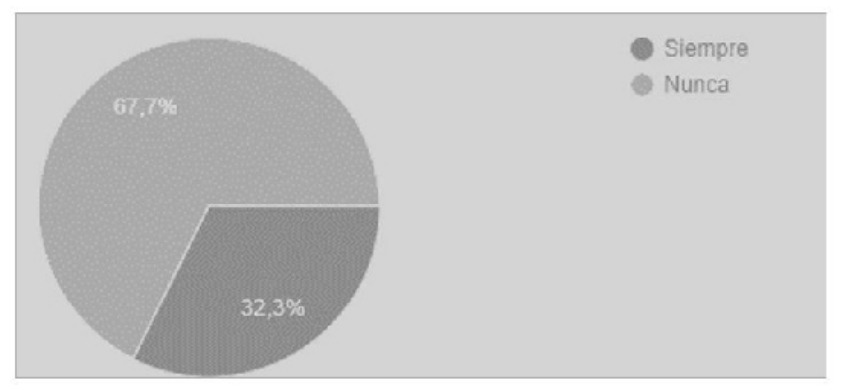

Figura 1. Hogares que presentan personas con problemas de alcoholismo.

Por otra parte, se evidenció que un $22 \%$ de los encuestados manifestaron que si hay 


\section{0}

miembros en su hogar que son consumidores de sustancias psicoactivas diferentes al alcohol y en este orden, un $5 \%$ de los encuestados manifestaron que también son consumidores continuos de sustancias psicoactivas diferentes al alcohol.

Del mismo modo, se evidencia que para la mayoría de los encuestados (60\%), es fácil adquirir bebidas embriagantes, en donde el $33 \%$ manifestaron que es muy fácil lograrlo y alrededor del $30 \%$ respondieron que algunas veces es fácil, el $26 \%$ respondieron que a menudo no es fácil y solo un $11 \%$ respondieron que es difícil adquirir bebidas embriagantes, de igual modo se evidencia que alrededor del $52 \%$ de los encuestados manifestaron que existen facilidades para adquirir sustancias diferentes al alcohol.

De igual modo, se obtiene que alrededor del $35 \%$ de los encuestados manifestaron que casi nunca han consumido bebidas embriagantes, alrededor del $33 \%$ manifestaron que algunas veces lo han hecho y alrededor del $22 \%$ nunca lo han hecho; pero aproximadamente el $10 \%$ de los encuestados son consumidores asiduos (siempre lo hacen), de bebidas embriagantes, igualmente podemos constatar que entre los que siempre consumen y algunas veces lo hacen, suman un $43 \%$ del total de encuestados.

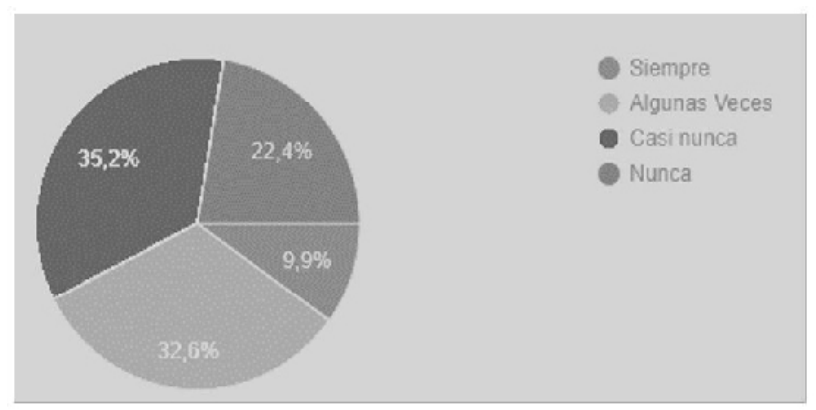

Figura 2. Distribución del consumo de bebidas embriagantes en los jóvenes entrevistados. Elaboración propia.

Finalmente, se constató que el rango de edad en el que la mayoría de los encuestados (27\%), manifestaron tener su primera experiencia con las bebidas alcohólicas está entre los 15 y 16 años, el segundo rango de edad reportado en la encuesta es entre los 13 y 14 años con un $25 \%$. Igualmente, se evidencia que solo alrededor del $15 \%$ manifestaron que no han probado bebidas alcohólicas y el $17 \%$ lo hizo entre los 17 y 19 años.

Se puede deducir que solo un $32 \%$ de los encuestados han postergado el tiempo para tener su primera experiencia con las bebidas alcohólicas. Igualmente, se evidencia que el rango de edad en el que generalmente se han presentado las experiencias con las SPA, es entre los 13 y 16 años. Estos resultados evidencian que no se distancian de los reconocidos por el gobierno nacional, al señalar que para el año 2016 el promedio de edad de inicio en el consumo fue de 13.6 años [22].

Para realizar la propuesta de gestión pública de prevención de sustancias psicoactivas en los jóvenes teniendo en cuenta un enfoque comunitario, es importante recordar que el consumo de SPA en los niños, niñas y adolescentes es una situación que afecta el desarrollo integral de la sociedad y a pesar de las diversas opiniones y acciones sobre el tema, son muy pocos los programas que con éxito han logrado prevenir paulatinamente este degradante fenómeno social que aparte de afectar emocionalmente a las familias $y$ las comunidades, incide en su desarrollo económico, productividad y bienestar general.

Por lo tanto, es necesario de los esfuerzos continuos de las autoridades destinados a reducir los elementos causantes de los riesgos y por otro lado, fortalecer una amplia variedad de elementos de protección por parte de padres de familia, comunidad y la escuela [23].

En razón a lo anterior y de acuerdo a lo conceptuado por [24] se propone un modelo de prevención con un enfoque social basado 
en modificar el entorno de desarrollo del individuo, más que en cambiar al individuo. Un modelo basado en la comunidad, en donde esta sea copartícipe del diseño de las acciones y coordinación del proceso, de tal forma que se empodere la participación ciudadana, entendida como gobernanza [25]. En donde el equipo coordinador conozca de primera mano los avances de la gestión de manera periódica y en donde cada uno de los programas que se desprenden del modelo se encuentren articulados y tengan direccionadas sus estrategias hacia el cumplimiento de las mismas metas y objetivos.

En este orden, la propuesta de prevención desde un enfoque comunitario debe iniciar con un diagnóstico que permita comprender a qué se está enfrentando realmente [26]. Desde dicho diagnóstico se desprenderá una serie de estrategias, actores, redes de apoyo, aspectos a intervenir y desde luego la importancia de la evaluación como elemento primordial que garantice generación de nuevas políticas que contribuyen al bienestar de los ciudadanos con soporte de legalidad [27].

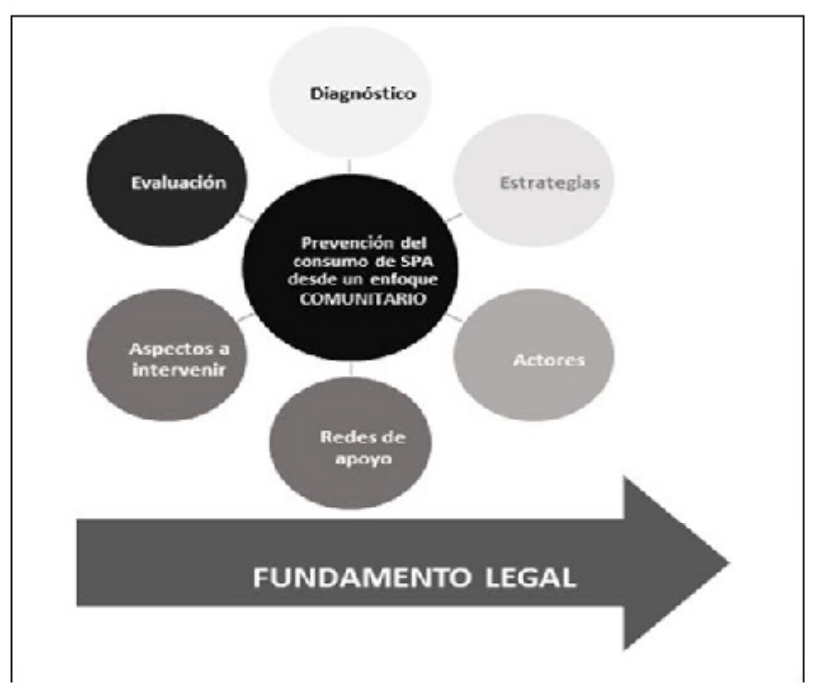

Figura 3. Proceso de gestión de la propuesta.

Conociendo que el objetivo general de dicha propuestaes prevenir el consumo de sustancias psicoactivas en los jóvenes del municipio, se tendrán en cuenta unas metas que permiten el logro de este y unas estrategias como acciones contundentes y concretas, dentro de estas acciones concretas se propone en primera medida la conformación del equipo base de trabajo, este equipo se caracterizan por la orientación de base y su miembros son los que asumen la mayor responsabilidad en la implementación del modelo, así mismo son los encargados de las acciones de monitoreo y análisis de la información recolectada periódicamente [28].

Otra estrategia relevante es la publicidad, como medio de impactar positivamente con cada uno de los programas y concientizar a los ciuadanos sobre la importancia de prevenir o evitar conductas que afecten el desarrollo social y saludable, especialmente a la juventud. Igualmente, la propuesta debe tener unas fuentes de financiación que garanticen recursos para el desarrollo e implementación de los programas con continuidad, por lo que es necesario la modificación del Estatuto de Rentas municipal para garantizar nuevos ingresos de uso propio, gestión ante entidades del orden nacional e internacional y establecimiento de alianzas como medio de articulación con el fin de que el ente territorial pueda potenciar sus capacidades [9].

Otras estrategias que deben ser tenidas en cuenta son el fortalecimiento de los programas deportivos y artísticos, minimizar tiempos sin supervisión de los adultos buscando que el joven se vincule activamente a las actividades benficiosas que le ofrece su entorno, sin dejar de lado las acciones gubernamentales encaminadas a mitigar las horas tarde de los jóvenes fuera de casa [29]. Igualmente, la implementación de programas comunitarios como por ejemplo institucionalizar días de cine o teatro al público que de un lado fomenten el aprecio a la cultura, el arte y de otro lado contribuyan a reducir los elementos causantes de los riesgos al tiempo que los padres puedan 


\section{2}

compartir con sus hijos momentos de sano esparcimiento [28].

Así mismo, el fortalecimiento de la parentalidad a través de progamas académicos y talleres continuos promovidos por las Empresas Administradoras del Plan de Beneficios en salud con intevención de la administración municipal a través de acciones que estimulen la participación de los padres de familia en las formaciones programadas. Finalmente, la continuidad y longitudinalidad, sobre todo en los equipos de salud púbica de la administración municipal y en las IPS, ya que la continua interacción de individuo y profesional de salud logran garantizar la resolución de muchos problemas de la población atendida [30].

Continuando con el desarrollo de la propuesta, es necesario tener en cuenta unos actores, entendidos como ese conglomerado que puede participar en el desarrollo de los programas generados a partir de la propuesta y que en algunos casos estarán en la capacidad de aportar recursos, ya que las alianzas permiten aprovechar efcientemente las ventajas que ofrece la union de fuerzas en la búsqueda de objetivos comunes, garantizando desarrollo e innovación en los programas [31]. Dentro de esos actores sobresalen las entidades del nivel nacional y departamental del estado, organismos de cooperación de gobiernos extrangeros, la OMS y sus diversos comités, la OEA y sus comisiones sobre el tema; dentro del sector privado podemos encontrar las diferentes organizaciones deportivas, los íconos representativos del arte y deporte y fundaciones empresariales dedicadas al trabajo social, quienes recíprocamente ayudan a la construcción de eslabones sólidos en la relación con su medio y en el fortalecimiento de su desarrollo [32].

Igualmente, es importante tener en cuenta a las organizaciones religiosas y confesionales, gremios de educadores e investigadores y demás profesionales de la salud [29]; ya que es importante fortalecer los valores sociales necesarios para empoderar acciones que contribuyan a tomar decisiones en base a la información reunida y no en los deseos [33].

Del mismo modo, se deben establecer e identificar unas redes de apoyo por un lado, las redes institucionales, conformadas por los miembros de los equipos de salud pública del municipio, la administración municipal, el personal de PyP de las IPS y agentes de las empresas administradoras del plan de beneficios de salud. De otro lado se encuentran las redes comunitarias, conformadas por todas las organizaciones comunitarias, tales como asociaciones de mujeres, asociaciones del adulto mayor, asociaciones de pequeños productores, cooperativas y las juntas de acción comunal.

De otro lado, se deben tener en cuenta los aspectos a intervenir al implementar la propuesta, estos son: En primer lugar el aspecto social que busca generar un entorno apropiado para el sano desarrollo de la juventud, aquí juegan un papel determinante las instituciones educativas y la alcaldía municipal, organizaciones comunitarias y demás instituciones de cultura y deporte tanto públicas como privadas; estos actores son los que a través de los programas implementados y la forma como se desarrollen dichos programas, contribuirán a generar atracción de los jóvenes hacia las prácticas deportivas, culturales y artísticas, alejándolos del riesgo del consumo [11].

Un segundo aspecto es el familiar, en este aspecto se intervienen las conductas inadecuadas para la formación de los jóvenes, se enfatiza en el control parental, buscando que los padres pasen mucho más tiempo con sus hijos. En este sentido, la escuela de padres debe enfilarse en generar conciencia y evidenciar la responsabilidad adquirida como 
padres o tutores. Este aspecto debe tener en cuenta el cuidado de los adolescentes, el control de las horas tarde y el cambio de actitud liberal de algunos padres [29].

El tercer aspecto es emocional, en este punto se busca que el joven definitivamente se aleje del riesgo del consumo, no por la acción de la prohibición, sino porque realmente encuentre atracción por los programas o actividades que le ofrece su entorno. En este orden, se debe empoderar el proyecto de vida del adolescente, que el joven desarrolle a través de sus actividades tanto académicas y deportivas como artísticas y culturales otras fortalezas como el trabajo en equipo, la disciplina, el fortalecimiento de la autoestima y el máximo esfuerzo para el logro de sus proyectos. [26].

Finalmente, la evaluación de cada uno de los programas que se desarrollan en el marco de la propuesta de gestión pública, deben estar sometidos a un proceso periódico de vigilancia, monitoreo y medición que permita evaluar resultados y nivel de impacto [25]. Para este proceso, se requiere diseñar indicadores específicos que faciliten la comparación y medición al tiempo que se ejecutan los programas.

Lo anteriormente expuesto, debe estar sobre la base de un fundamento legal que brinde estabilidad jurídica a cada uno de los programas, que responda a los mandatos refrendados en la Constitución nacional y a los convenios internacionales avalados y aceptados en esta materia. En razón a lo anterior, la Constitución Nacional [34] en su artículo 49 establece que el Estado debe garantizar la salud y el saneamiento ambiental a los ciudadanos y que se debe propender para que las personas tengan acceso a los servicios de promoción, protección y recuperación de la salud.

De la anterior se desprenden muchas otras normas que buscan reglamentar el mandato constitucional, sobresalen la Ley 1098 de 2006 , del Senado de la república [35] conocido como el Código de infancia y adolescencia cuya finalidad es garantizar un desarrollo pleno y armonioso de los Niños, niñas y adolescentes, además de promover la protección integral de esta población. En su orden, la Ley 1955 de 2019 del Congreso de la república [36] en su Artículo 206 establece la política de atención integral a la primera infancia, la infancia y adolescencia en cuanto a la formulación e implementación de políticas relacionadas con la prevención de la violencia y el consumo de SPA. Igualmente, la resolución 0089 del 16 de enero de 2019, emanada del ministerio de salud por medio de la cual se adopta la política integral para la prevención y atención del consumo de SPA [37].

\section{Conclusiones}

Es evidente que el consumo de sustancias psicoactivas en los jóvenes, afecta negativamente el capital social ya que este se encuentra estrechamente relacionado con la consolidación del tejido social [8]. Por lo tanto, a parte del estancamiento del desarrollo que pueda llegar a presentar una comunidad afectada por el consumo, se demandan recursos importantes que deben ser invertidos en la recuperación de los afectados y no en otras necesidades relevantes que bien podrían ser priorizadas si no existiera este problema. En este orden, es evidente que el capital social se constituye en una perspectiva que promueve la participación colectiva de una sociedad con el fin de lograr objetivos de desarrollo [38].

Autores como [14] afirman que el objetivo de la nueva gestión pública es lograr mayor eficiencia en las Entidades en cuanto a hallar alternativas de solución a las demandas sociales, teniendo como base la inclusión ciudadana en la toma de decisiones y dando como resultado las políticas públicas las 


\section{4}

cuales siempre deben estar en la dirección de la mejora continua. Por lo tanto, es importante que la comunidad participe activamente en la generación de propuestas de gestión y políticas públicas para su propio beneficio, es evidente que su involucramiento activa el empoderamiento y la corresponsabilidad en cada una de las acciones, lo cual conlleva a generar mayor efectividad en los resultados y mayor índice de desarrollo.

Estapropuestadegestión públicadeprevención del consumo de sustancias psicoactivas en jóvenes, con enfoque comunitario es una idea innovadora en la región, ya que siempre se ha actuado referente al tema del consumo desde una óptica de la prohibición hacia los jóvenes, desde la acción coercitiva, siempre tratando de cambiar al individuo, sin contemplar la posibilidad de cambiar el entorno en donde se desarrolla el individuo [11]. Siendo el anterior, uno de los elementos primordiales sobre los que se desarrolla esta propuesta.

El incremento del consumo de SPA es un fenómeno que ha afectado a muchas personas en el mundo sin excepción de nuestro país, en donde los estudios han demostrado notorio aumento del consumo, principalmente en la población joven y adolescente quienes por su etapa de desarrollo demuestran vulnerabilidad ante esta situación [2]. Los estudios en América Latina, han demostrado una marcada tendencia hacia el consumo del alcohol principalmente en la población joven, siendo Colombia uno de los países que más ha demostrado prevalencia del consumo en los estudiantes de secundaria. En cuanto al consumo de otras sustancias como cocaína, se evidencia incremento del consumo en jóvenes en edad escolar.

Las regiones de Colombia afectadas por el conflicto armado, registran escasez de investigaciones referentes al consumo de SPA en jóvenes y adolescentes; sin embargo, algunos estudios evidencian inicios del consumo a edades más tempranas que las del promedio nacional [5].

Lo anterior, debido a presencia de diferentes actores, entre ellos los grupos armados que involucran movimiento de personal entre diferentes regiones que traen consigo la práctica del consumo de SPA, relacionado por algunos padres de familia con una percepción de inseguridad, fenómeno que pasa desapercibido para los jóvenes, ya que estos son receptivos a la convivencia con pares que han sido víctimas del conflicto [39].

La investigación evidenció que en la mayoría de hogares de los entrevistados existen personas adultas con serios problemas de consumo de alcohol, lo cual podría tener alguna relación con el consumo de bebidas embriagantes manifestado por un alto porcentaje de los encuestados. Caso diferente, en lo referente al bajo porcentaje de entrevistados que manifestaron tener en sus hogares personas consumidoras de sustancias diferentes al alcohol, lo cual podría tener alguna relación con el bajo porcentaje de entrevistados que manifestaron consumir sustancias psicoactivas diferentes al alcohol. Igualmente, para los jóvenes que participaron en el estudio es muy fácil adquirir bebidas embriagantes y medianamente fácil acceder a sustancias diferentes al alcohol, esto podría demostrar por qué los jóvenes se están iniciando en el consumo de bebidas embriagantes entre los 13 y 16 años.

Es necesario implementar una propuesta de gestión pública que permita prevenir el consumo de sustancias psicoactivas en los adolescentes del municipio de Toledo, para lo cual es importante iniciar con un diagnóstico detallado de la situación, desarrollar programas que se articulen entre sí e involucrar a la comunidad de manera activa en cada uno de los procesos [23]. Para la implementación 
de esta propuesta, se deben tener en cuenta todos los actores internacionales, nacionales y locales, tanto públicos como privados que puedan aportar recursos, conocimiento y experiencia al desarrollo exitoso de cada uno de los programas de los que requiere el desarrollo del objetivo de la política.

\section{Referencias}

[1] UNODC, "Informe Mundial Sobre las Drogas 2019”, 2019. [En línea]. Disponible en: https:// wdr.unodc.org/wdr2019/prelaunch/WDR2019_ B1_S.pdf

[2] OEA-CICAD, "Informe Sobre el Consumo de Drogas en las Américas 2019”, Organización de Estados Americanos, Washington, 2019

[3] M.d.P. Reyes, J.A. Alvarez y C. Rosales, Prevención de la Violencia, consumo de Drogas y otras conductas de Riesgo, V.G. Gutierrez Campos y A.J.M. Orozco, Edits., Ciudad de México: Centros de Integración Juvenil, 2016

[4] OPS, “Indicadores Básicos 2019: Tendencias de la Salud en las Américas", Washington DC, 2019

[5] C. Gantiva, H.H. Ramirez, A. Angarita, A. Parado y A. Guillen, "Consumo de sustancias psicoactivas (SPA) en una region posconflicto armado", Revista Colombiana de Psiquiatría, vol. 41, no. 2, pp. 299-308, 2012

[6] A. Bacca, "La educación no formal contextualizada: huellas de su desarrollo y transformación en Cúcuta", Educación y Humanismo, vol. 16, no. 27, pp. 70-86, 2014

[7] J. Durston, Serie políticas sociales ¿Qué es el capital social comunitario?, Santiago de Chile: CEPAL/Naciones Unidas, 2000, p. 45
[8] N.D. Bravo, "El capital social como determinante de salud pública" Revista de Calidad Asistencial, vol. 32, pp. 346-346, 0209 2017

[9] J. Barrena-Martinez, L. Cricelli, E. Ferrandiz, M. Greco y M. Grimaldi, "Fuerzas conjuntas: hacia una integración de la teoría del capital intelectual y el paradigma de innovación abierta", Journal of Business Research, vol. 112, pp. 261-270, 29 Octubre 2019

[10]Y. Pérez Rodríguez, y A. Coutín Domínguez, "La gestión del conocimiento: un nuevo

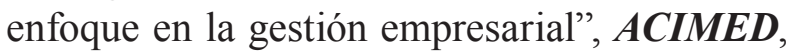
vol. 13 , no. 6,2005

[11]A.C. Duarte-Rodríguez, "Habilidades gerenciales y su importancia para el éxito de una organización”, Trabajo de grado, Universidad Militar Nueva Granada, 2013

[12] A. Luque, V. E. Melino y P. R. Solís, "Gestión pública socialmente responsable: Caso hilando el desarrollo en Ecuador" Revista Venezolana de Gerencia, vol. 24, no. 2, pp. 285-307, Noviembre 2019

[13] J. Hernandez, Y. F. Hernandez, M. Gil y H. Cárdenas, "Evaluación del modelo integrado de planeación y gestión (MIPG) en las entidades territoriales del Estado colombiano", Aglala, pp. 443-463, Septiembre 2018

[14] D. J. De la Garza, E. R. Yllán y D. Barredo, "Tendencias en la administración pública Moderna: La nueva gestión pública en México" Revista Venezolana de Gerencia, vol. 23, no. 81, pp. 31-48, Enero 2018

[15] L. Morris, O. Salazar y E. Barrientos, "Sensitivity analysis for decision making in the context of production", International congress on project management and engineering, pp. 2080-2091, 2019 
[16] A. Alvarez- Collazos y S. Chica-Vélez, Gestión de las organizaciones públicas, Bogota: Escuela Superior de Administración Pública, 2008

[17] G. Buitrago Olivares, "Estrategia de gestión escolar para la prevención del consumo de drogas en adolescentes de la Básica y Media del Colegio Costa Rica IED Bogotá D.C”, trabajo de grado, Universidad Libre, 2015

[18] Dirección Nacional de Planeación, "Terridata," 2019. [En línea]. Available: https://terridata. dnp.gov.co/index-app.html\#/acercade. [Último acceso: Septiembre 2019]

[19] ODC, "Estudio Nacional de Consumo de Sustancias Psicoactivas en Población Escolar Colombia - 2016”. Bogotá, 2016

[20] ODC, "Reporte de Drogas de Colombia 2017", Legis S.A, Bogotá, 2017

[21] Alcaldía Municipal de Toledo, "Unidos para Avanzar 2016-2019”, Toledo (N de S), 2016

[22] K. Jimenez Meriño y T.M. Rojas Fernández, "Consumo de sustancias en estudiantes de 12 a 18 años en la ciudad de Barrancabermeja", trabajo de grado, Universidad Cooperativa de Colombia, 2018

[23] I.D. Sigfusdottir, T. Thorlindsson, A.L. Kristjansson, K.M. Roe y J.P. Allegrante, "Prevención del uso de sustancias para adolescentes: el modelo islandés" Health Promotion International, vol. 24, no. 1, pp. 1625, 11 Diciembre 2008

[24] L. Guillen Pereira, N.P. Arma Castañeda, A.A Formoso Mieres, J.R. Guerra Santiesteban, D.F. Vargas Vera y A. Fernández Lorenzo, Angie, "Estrategia pedagógica para reducir y prevenir el consumo de drogas desde la Educación Física", Revista Cubana de Investigaciones
Biomédicas, vol. 36, no. 2, pp. 111-126, 2017

[25] A. Valdez, "Paradigmas emergentes en la gestión pública en América Latina", Revista Venezolana de Gerencia, vol. 24, no. 86, pp. 325-339, 20 Febrero 2019

[26] Facultad de Medicina UC, [Archivo de Video], 2019.

[27] A.J. Sanchez, A. Melian y E. Hormiga, "El concepto de capital intelectual y sus dimensiones" Investigaciones Europeas de Dirección y Economía de la Empresa, vol. 13, no. 2, pp. 97-111, 2007

[28] Y. Tunjano Gutierrez, "La cultura ciudadana como eje transversal de la convivencia y seguridad ciudadana", trabajo de grado, Universidad Católica Colombiana, 2014

[29] G.S Ríos Niño, “Utilización del tiempo libre como factor y factor de riesgos en niños y adolescentes de 9 a 18 años de la i.e.d serrezuela del municipio de madrid", trabajo de grado, Universidad de Cundinamarca, 2018

[30] R. Pastor, A. López, M. Pérez y J. Gérvas, "Continuidad y longitudinalidad en medicina general en cuatro paises europeos", Revista española de salud pública, vol. 71, no. 5, pp. 479-485, Septiembre 1997

[31] J.J. Gonzalez y L. Alvarez, "Gestión de Conocimiento e Innovación Abierta: hacia la conformación de un modelo teórico relacional", Revista Venezolana de Gerencia, vol. 24, no. 88, pp. 1199-1222, 2019

[32] E. Rodríguez, Políticas públicas y marcos legales para la prevención de la violencia relacionada con adolescentes y jóvenes Políticas públicas y marcos legales para la prevención de la violencia relacionada con adolescentes y jóvenes Estado del arte en 
América Latina 1995-2004, Perú: Cooperación Técnica Alemana-GTZ

[33] Oficina de las Naciones Unidas contra la Droga y el Delito Viena, ESCUELAS Educación de base escolar para la prevención del uso indebido de drogas, Nueva York, NACIONES UNIDAS, 2004

[34]Senado de la República, "Constitución Política de Colombia”, 2309 2019. [En línea]. Available: http://www.secretariasenado.gov.co/senado/ basedoc/constitucion_politica_1991.html. [Último acceso: 0610 2019]

[35] Secretaría Jurídica Distrital de la Alcaldía Mayor de Bogotá, "Ley 1098 de 2006 -Código de Infancia y Adolescencia", 08 Noviembre 2006. [En línea]. Available: https://www. alcaldiabogota.gov.co/sisjur/normas/Norma1. jsp?i=22106. [Último acceso: 08 Octubre 2019].

[36] Congreso de la República de Colombia, Ley 1955 de 2019, Plan Nacional de Desarrollo 2019-2022 "Pacto Por Colombia Pacto Por la Equidad”,» 25 Mayo 2019. [En línea]. Available: https://dapre.presidencia.gov.co/normativa/ normativa/LEY\%201955\%20DEL\%2025\%20 DE\%20MAYO\%20DE\%202019.pdf

[37] Ministerio de Salud y Protección Social, "Resolución 00089 del 16 de Enero de 2019”, 16 Enero 2019. [En línea]. Available: https://www.minsalud.gov.co/sites/rid/Lists/ BibliotecaDigital/RIDE/DE/DIJ/resolucion089-de-2019.pdf.

[38] J.A. Avalo, J.L. Yagüe y G. Cangahuala, “E1 capital social y la planificación adaptativa en una comunidad industrial innovadora del Perú", Revista de Estudios Gerenciales, vol. 32, pp. 162-169, 2016
[39] F. Simanca, M. Unriza, H. Llanez y J. Paez, "Desafíos para la construcción de una cultura de paz desde la escuela", Aglala, pp. 303-318, Agosto 2019 